\title{
DÉTECTION ET ANALYSE AUTOMATIQUE des aérosols atmosphériques par lidar infrarouge
}

\author{
Jean-Pierre CARIOU \\ Ludovic THOBOIS \\ LEOSPHERE \\ jpcariou@leosphere.com
}

Le changement climatique récent est attribué entre autres à l'impact des particules atmosphériques d'origine naturelle ou des activités humaines d'après le dernier rapport du GIEC [IPCC, Climate Change 2014: Synthesis Report]. Les aérosols affectent également les propriétés des nuages et influencent le cycle de l'eau, pouvant intensifier les moussons. En avril 2010, le volcan Eyjafjallajökull a créé un panache de plusieurs milliers de kilomètres sur l'Europe et causé la fermeture de plusieurs aéroports internationaux, clouant au sol des millions de voyageurs et causant une perte économique globale de 1,7 milliard de dollars. Tous les jours, les aérosols transfrontaliers (panaches de sable du Sahara, résidus de combustion des forêts canadiennes) affectent la santé des populations habitant notamment en zones urbaines ainsi que les activités économiques.

du changement de polarisation ou de fréquence permet une mesure résolue en distance de la concentration des différentes composantes de l'atmosphère, de leur vitesse et de la localisation des nuages et couches d'aérosols.

Deux grands principes sont utilisés. Le premier utilise la détection directe : le signal est proportionnel à la puissance optique collectée, intégrée dans une bande spectrale et un angle solide. Le second utilise la détection cohérente (ou hétérodyne) : par interférométrie, le signal reçu reproduit le champ du signal optique, et donc son amplitude, sa phase et sa fréquence, donnant accès en plus à la mesure de la vitesse du vent. Chaque principe utilise une technologie adaptée avec ses caractéristiques, ses avantages et ses inconvénients. Pour illustrer les principes et les performances de ces lidars, nous considérons deux exemples d'instruments : - un ceilomètre (ou télémètre nuage) infrarouge à détection directe à 0,91 um, tel que le CL51 de Vaisala; un lidar IR à 1,54 $\mu$ m à détection cohérente, tel le WINDCUBE200S de Leosphere.

Le tableau 1 indique les caractéristiques des deux instruments.

\section{Localisation des nuages et des aérosols}

\section{Principes}

Pour le ceilomètre à détection directe, les signaux sont inversés par plusieurs algorithmes dont l'algorithme d'inversion de Fernald-Klett [2] afin de calculer les paramètres optiques de chaque couche d'aérosols : coefficient d'extinction, de rétrodiffusion, épaisseur optique.

La voie de détection élastique du CL51 mesure le signal rétrodiffusé sur toutes les polarisations [3]. D'autres lidars plus complexes mesurent le signal séparément sur les deux polarisations. Le rapport de dépolarisation informe 
alors sur la sphéricité des particules et permet de distinguer les cristaux de glace des gouttelettes, ou les nuages de sable des nuages d'eau.

Pour le lidar cohérent IR, en plus de la mesure de vitesse du vent (voir plus loin), la puissance du signal rétrodiffusé est également traitée pour détecter les différentes couches de l'atmosphère, qu'elles soient constituées de nuages ou d'aérosols. Pour la caractérisation de la couche convective, les mesures de vent sont aussi utilisées.

\section{Détection des structures}

Couche limite atmosphérique

La couche limite atmosphérique (CLA) représente la couche basse de l'atmosphère en interaction turbulente avec le sol. Elle concentre la majeure partie des aérosols et des polluants. Son épaisseur varie selon l'heure de la journée, de la saison et de la latitude du lieu, entre quelques dizaines de mètres de nuit sur l'océan à plus de $3 \mathrm{~km}$ de jour aux tropiques. Au sommet de la CLA, la

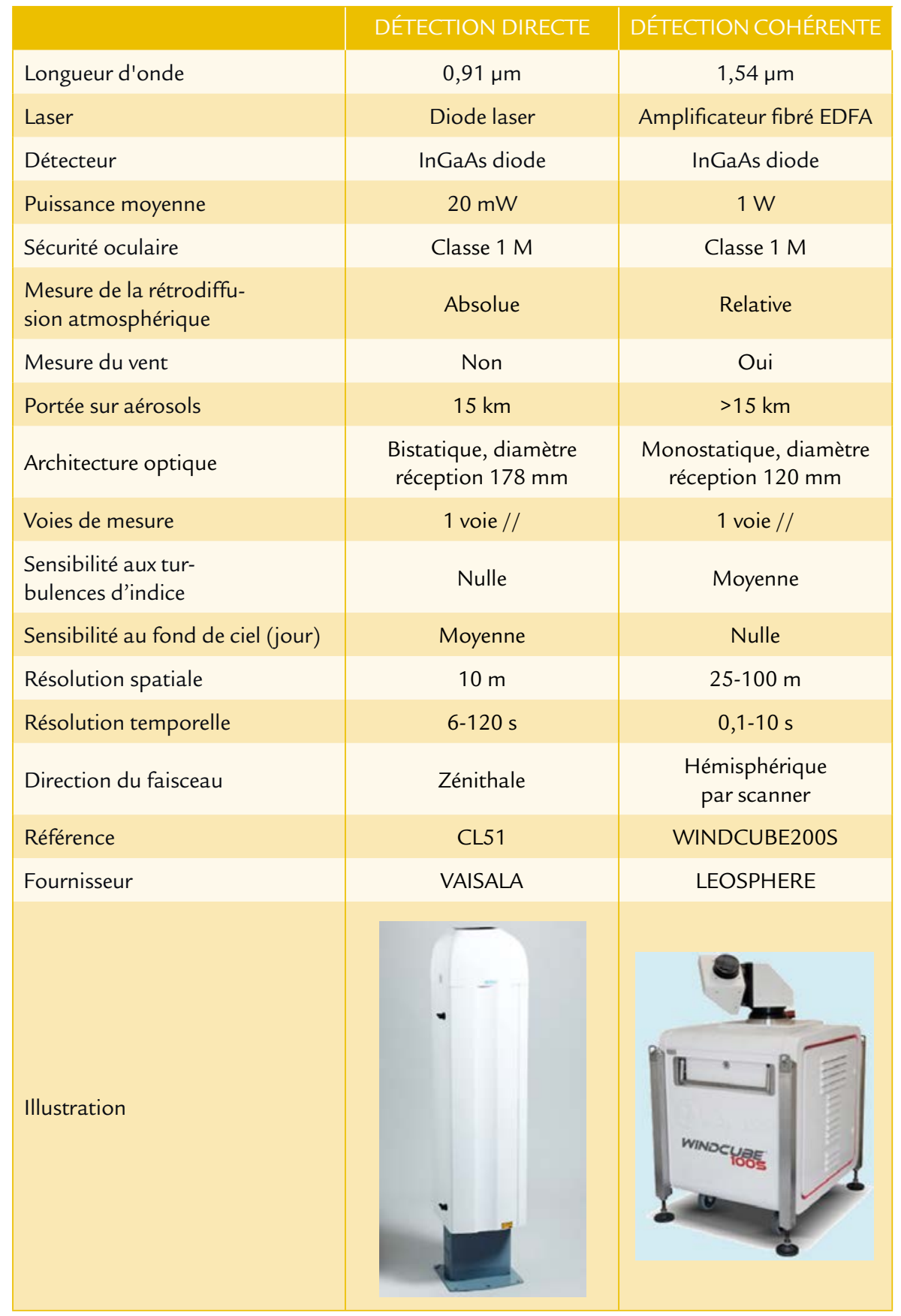

Tableau 1. Caractéristiques des lidars IR.

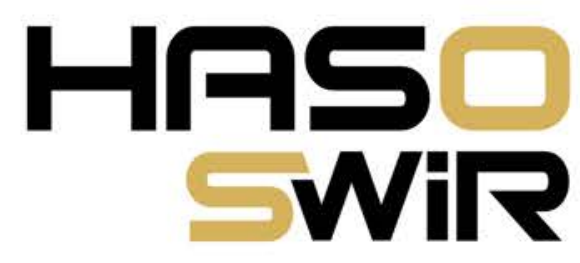

New Shack-Hartmann

wavefront sensor for

Short-Wave Infrared

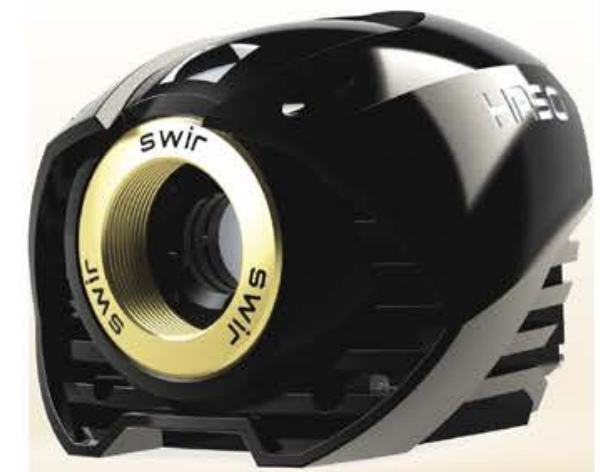

Wavelength range $0.9-1.7 \mu \mathrm{m}$ Absolute accuracy
Lambda/100

Acquisition Up to $99 \mathrm{~Hz}$

Compact and versatile

Contact us for more details: contact@imagine-optic.com or +33164861560 


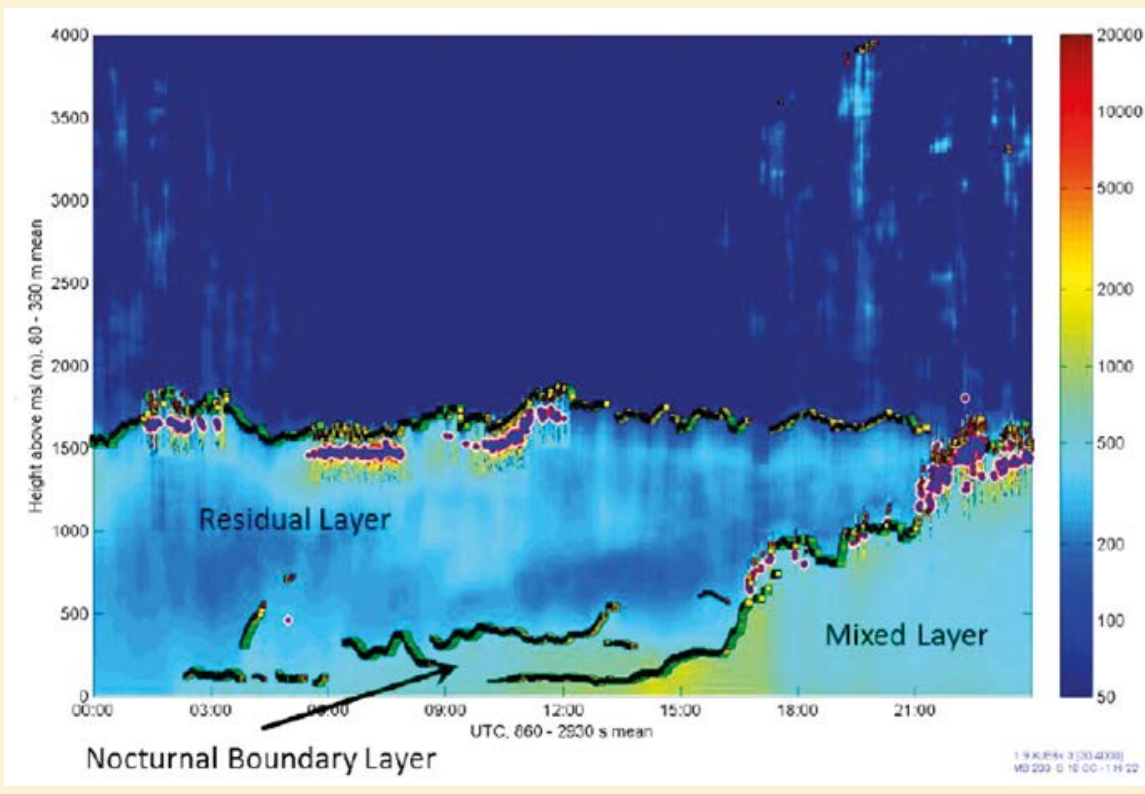

Figure 1. Observation du cycle de la couche limite atmosphérique par le ceilomètre (observatoire de Chibolton). turbulence augmente et la concentration des aérosols chute brusquement. Pour détecter ce sommet, l'algorithme de détection des lidars IR analyse le gradient vertical du signal élastique sur la voie parallèle et sa continuité au cours du temps.

La figure 1 montre un exemple de détection par le CL51 de la CLA. On observe un cycle typique de couche limite: pendant la nuit, la couche nocturne varie entre 300 et $400 \mathrm{~m}$. Elle est surmontée par une couche résiduelle qui s'étend jusqu'à $1500 \mathrm{~m}$ de hauteur. Après $9 \mathrm{~h}$ (UTC), la CLA augmente progressivement par la convection progressive et atteint son maximum à $1500 \mathrm{~m}$ vers $21 \mathrm{~h}$.

Spécifiquement pour le lidar IR cohérent, la mesure du vent vertical à haute fréquence typiquement toutes les secondes permet de mesurer efficacement le sommet de la couche limite convective.

\section{Nuages et couches d'aérosols}

L'étape suivante est la détection de la base et du sommet des nuages et des couches d'aérosols. La méthode utilise également un seuillage automatique sur le gradient du rapport de rétrodiffusion atténué [6]. La figure 2 montre la détection de la base des nuages sur le CL51.

Les quatre étapes de l'algorithme appliqué aux signaux du lidar cohérent IR sont décrites en figure 3. Entre le lidar à détection directe et le lidar cohérent, la différence de résolution spatiale n'est guère sensible à l'échelle de la portée. Les performances de détection et localisation des nuages sont très voisines. La faible diffusion moléculaire aux longueurs d'onde proche IR favorise le contraste de la frontière de la CLA.

\section{Mesure des paramètres optiques des aérosols}

Une fois les structures détectées, les paramètres optiques sont mesurés pour chaque couche : extinction optique $\alpha_{a}$, coefficient de rétrodiffusion, épaisseur optique AOD.

Le coefficient de rétrodiffusion atténué correspond au signal reçu, corrigé par la réponse en distance du lidar.

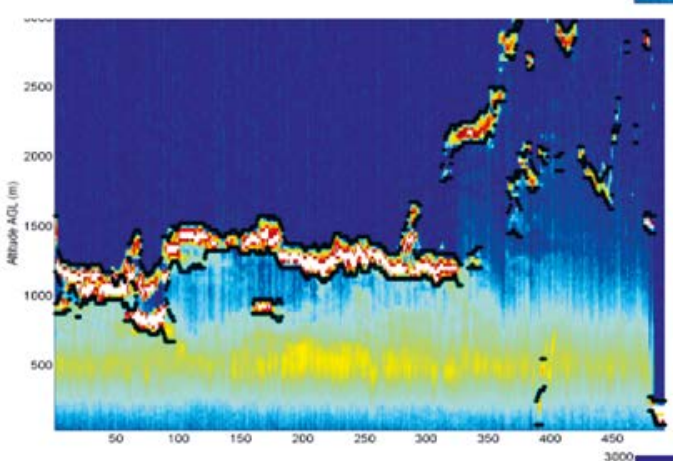

b) Vaisala Ceilometer CL51 Backscatter: 2013/11/24

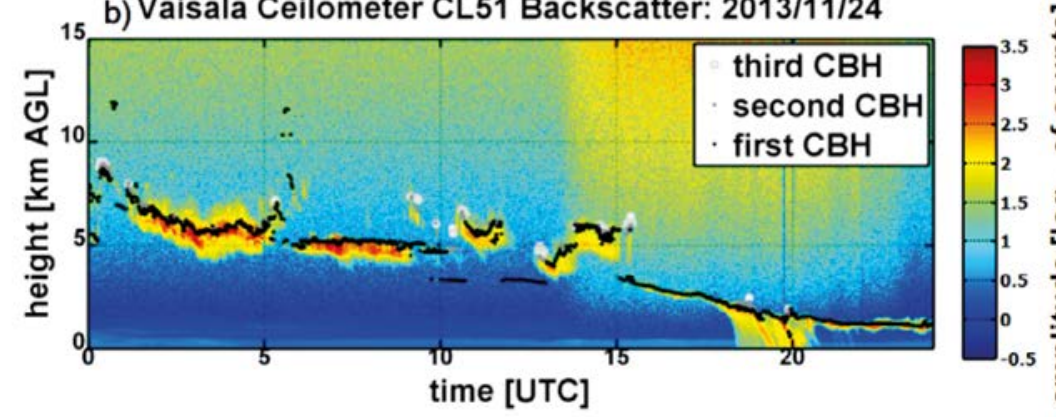

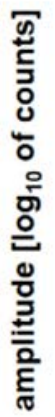

\section{Etape 4 : Base et \\ sommet superposés au CNR au CNR}

Figure 2. Détection automatique de la base des nuages par le CL51. 
La conversion du signal optique en coefficient de rétrodiffusion se fait par étalonnage initial sur une cible calibrée en albedo. Alors que sur un lidar bistatique à détection directe la décroissance du signal est quadratique avec la distance en dehors des courtes distances où le recouvrement émission et réception est partiel, la dépendance est plus complexe sur un lidar cohérent, sensible à la courbure de l'onde et aux défauts de phase dus à la turbulence d'indice. Le protocole de mesure du lidar cohérent inclut donc une mesure initiale de cette fonction instrumentale par un tir horizontal dans une couche atmosphérique homogène. Par combinaison avec l'épaisseur optique, il est alors possible de calculer le coefficient de rétrodiffusion absolu atténué.
L'étape future est de convertir les données optiques en concentration massique, grâce à la connaissance des propriétés microphysiques des particules, recueillies in situ.

\section{Classification des aérosols et des nuages}

Le rapport lidar, entre le coefficient d'extinction et le coefficient de rétrodiffusion, et le rapport de dépolarisation particulaire (DR), quand il est mesuré, sont finalement utilisés pour classifier automatiquement les aérosols en différentes catégories [7]: pollution continentale, aérosols marins, poussières et cendres volcaniques. Les nuages sont classés en quatre catégories : cirrus, altostratus,

\section{CNR}

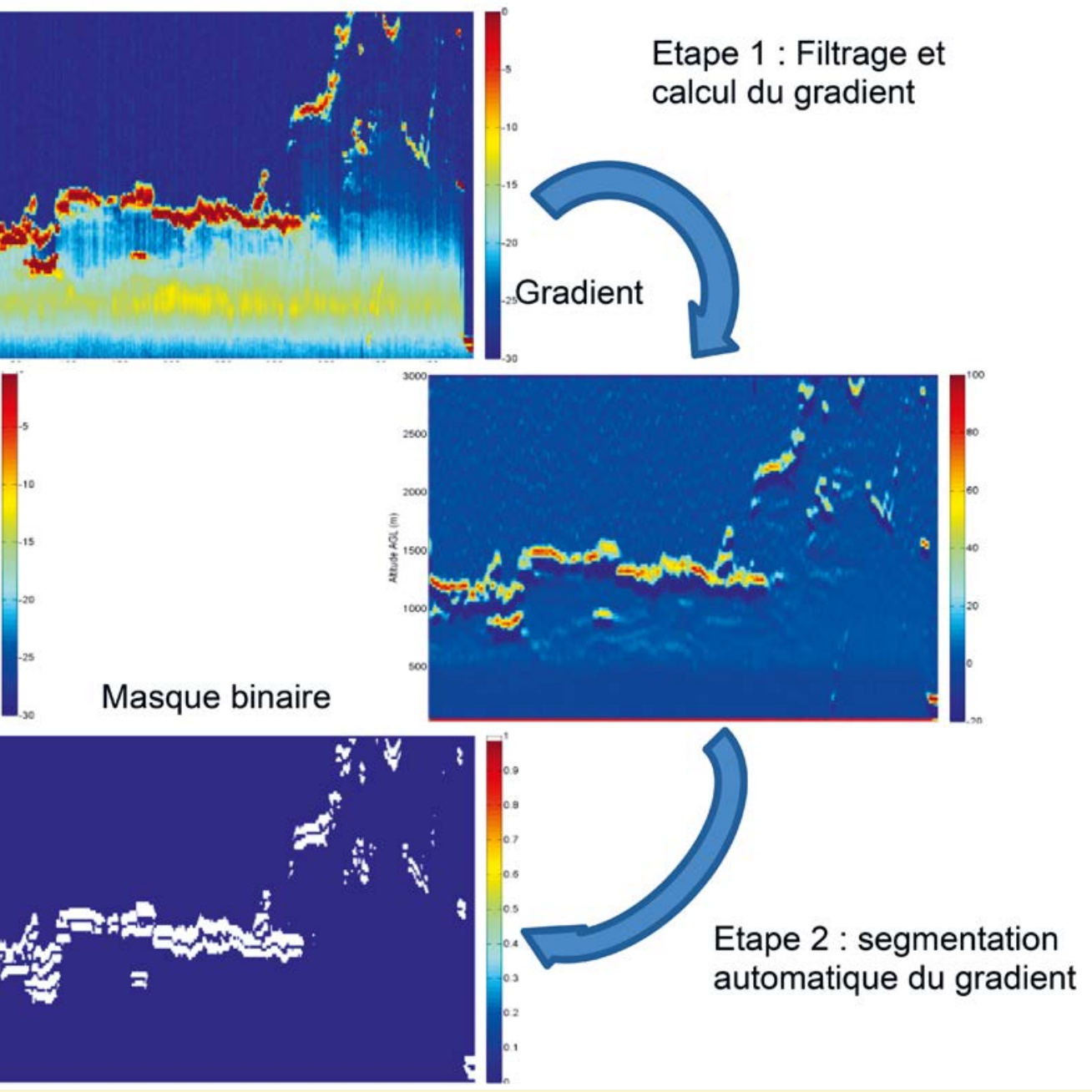

Figure 3. Détection automatique en 4 étapes de la base et du sommet des couches atmo-sphériques sur un lidar cohérent IR.

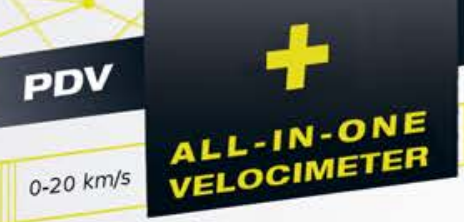

VISAR

$0.2 \mathrm{~km} / \mathrm{s}$

AELOCIMETEF

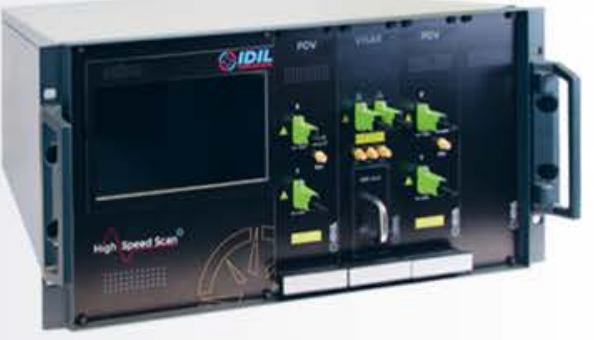

Get the best results

in shock-physics studies

4 spead resolution

8 Time resolution

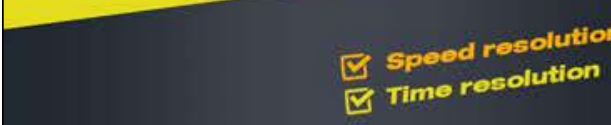

(1)

$X=$ Time $(\mu s): Y=$ Speed $(\mathrm{m} / \mathrm{s})$

$\mathrm{P}=$ Precurso

Features

- Modular and compact velocimeter

- 1-4 PDV / VISAR channels

- Faster fiber-alignment system

- User-friendly touchscreen interface

- High speed measurement

- Absolute speed measurement

- Elastic precursor wave detection (P)

- Projectile / dust detection

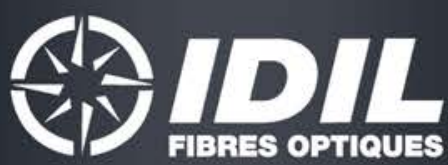




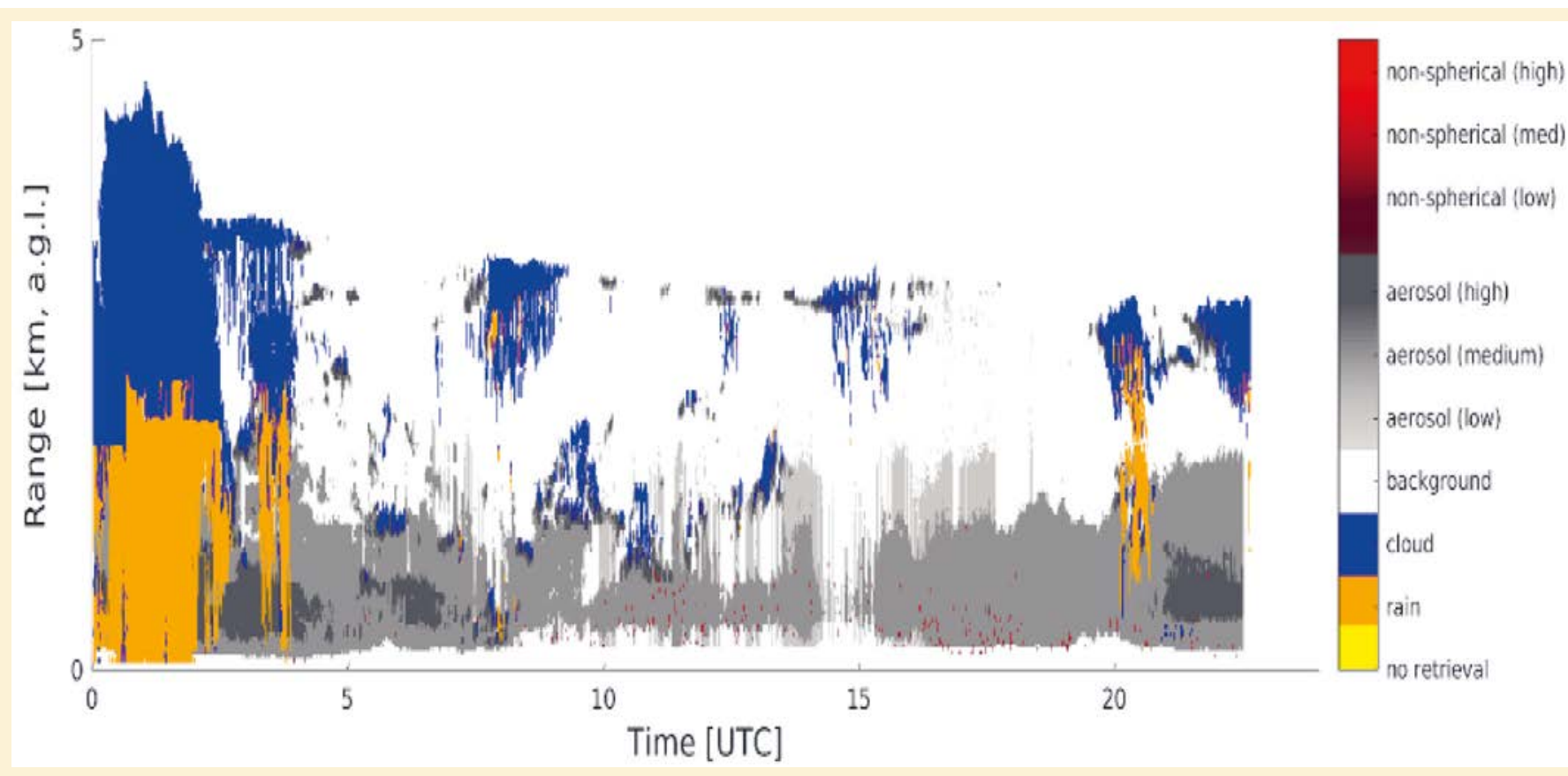

Figure 4. Classification des nuages et des aérosols avec le ceilomètre.

altocumulus, nuages bas, à partir de leur altitude et de leur épaisseur optique (figure 4).

\section{Mesure de la vitesse du vent}

\section{Principe}

Le lidar IR à détection cohérente émet des impulsions brèves et monofréquences dans l'atmosphère. Le signal détecté après rétrodiffusion sur les aérosols de l'atmosphère est mélangé dans un interféromètre à une fraction de la source maître (oscillateur local) (figure 5).

Le signal de battement, par effet Doppler, possède une fréquence proportionnelle à la vitesse radiale des aérosols (vitesse du vent projetée sur la ligne de visée) et une puissance proportionnelle au coefficient de rétrodiffusion atténué. Le traitement d'analyse spectrale par un processeur FPGA temps réel, suivi d'une estimation spectrale par MLE (maximum likelyhood estimator) produit la vitesse radiale du vent, sa dispersion, et l'intensité du signal. Pour retrouver les trois composantes du vecteur vent, une reconstruction est réalisée à partir de visées séquentielles dans 3 à 5 directions non coplanaires.

\section{Architecture optique}

Issu d'une souche technologique transférée de l'ONERA/DOTA, le lidar WINDCUBE200S utilise une chaîne laser MOPA (master oscillator power amplifier) comprenant un oscillateur local continu semi-conducteur à $1550 \mathrm{~nm}$ et un amplificateur fibré en mode impulsionnel. La résolution spatiale verticale de 50 à $100 \mathrm{~m}$ est associée à une durée d'impulsion de 200 à 400 ns (tablean 1). L'ouverture du télescope est limitée à $150 \mathrm{~mm}$ par la cohérence transverse du faisceau après propagation dans l'atmosphère turbulente.

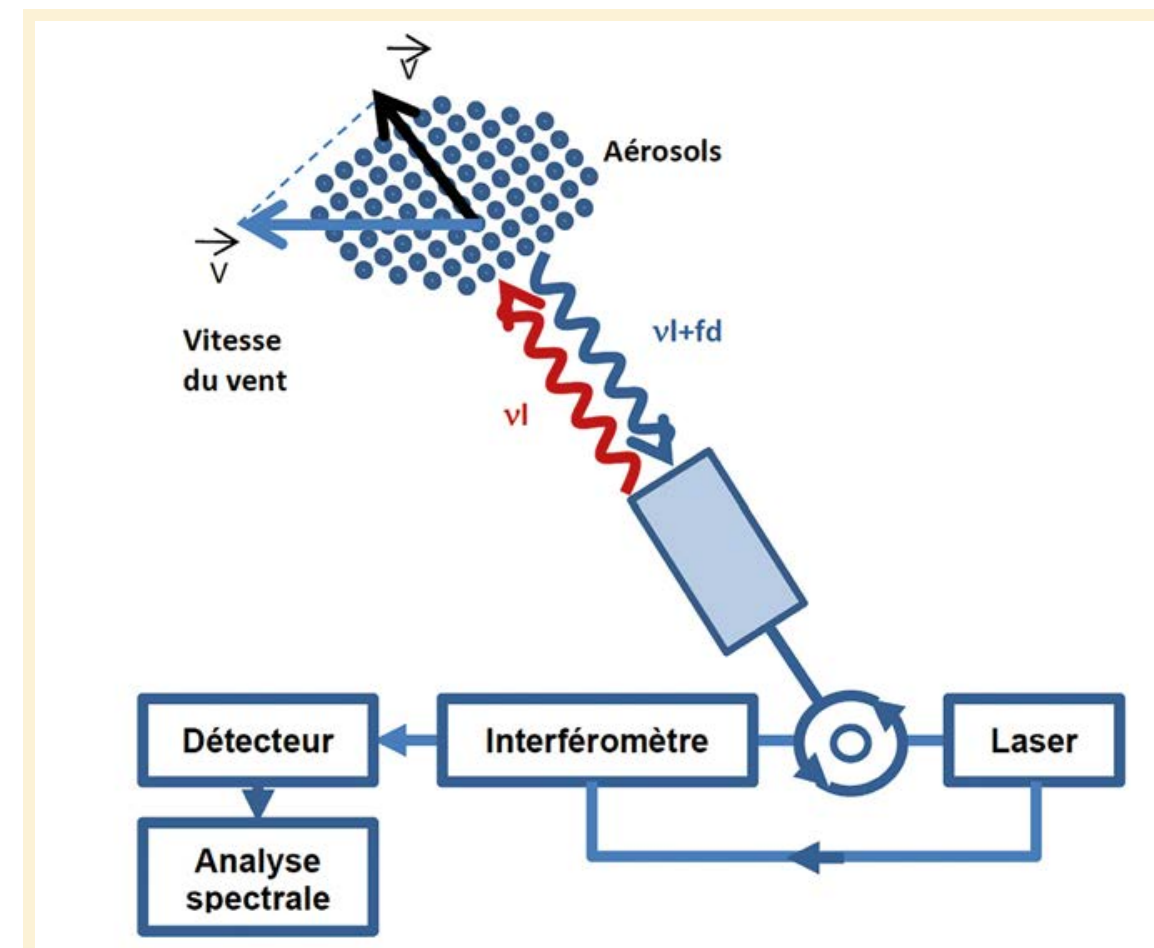

Figure 5. Schéma de principe du lidar doppler cohérent. 
Dans l'instrument tous les composants sont fibrés, conférant une grande souplesse d'architecture et une forte robustesse aux perturbations.

\section{Cas du volcan islandais d'avioil 2010}

En avril 2010, le volcan Eyjafjallajökull a projeté dans le ciel européen des milliers de tonnes de poussières. Une partie de l'espace aérien européen a été alors fermée de façon préventive, occasionnant de lourdes pertes économiques. Faute de données d'observation disponibles, les avis de cendres émis par le centre anglais en charge du suivi du panache volcanique reposaient alors quasiment exclusivement sur des modèles de dispersion. Suite à cette sérieuse alerte, la France s'est équipée en 2016 d'un réseau de 5 lidars à détection directe. Un sixième, mobile, sera installé à Toulouse mais pourra être déplacé en cas de crise volcanique. Ils alimenteront le modèle de dispersion atmosphérique de Météo-France MOCAGE. Au Royaume-Uni, le réseau Lidarnet opéré par le MetOffice possède 40 ceilomètres à détection directe dans le proche IR, version courte portée des lidars troposphériques.

Le lidar cohérent Windcube $200 \mathrm{~S}$ a également mesuré le nuage de

\section{LEOSPHERE}

LEOSPHERE est une société française créée en 2004, appartenant au groupe VAISALA depuis 2018. Située à Saclay (91), elle compte aujourd'hui 125 collaborateurs, dont 35 en R\&D. Avec une expertise des lidars atmosphériques, elle réalise un chiffre d'affaire de $28 \mathrm{M} €$ (2018), dont $90 \%$ à l'export. La technologie est issue d'un transfert de I'ONERA/DOTA. Avec ses lidars de mesure d'aérosols et de vitesse du vent, LEOSPHERE adresse les marchés de l'énergie éolienne, de la météorologie, de la qualité de l'air et de la sécurité aéroportuaire.

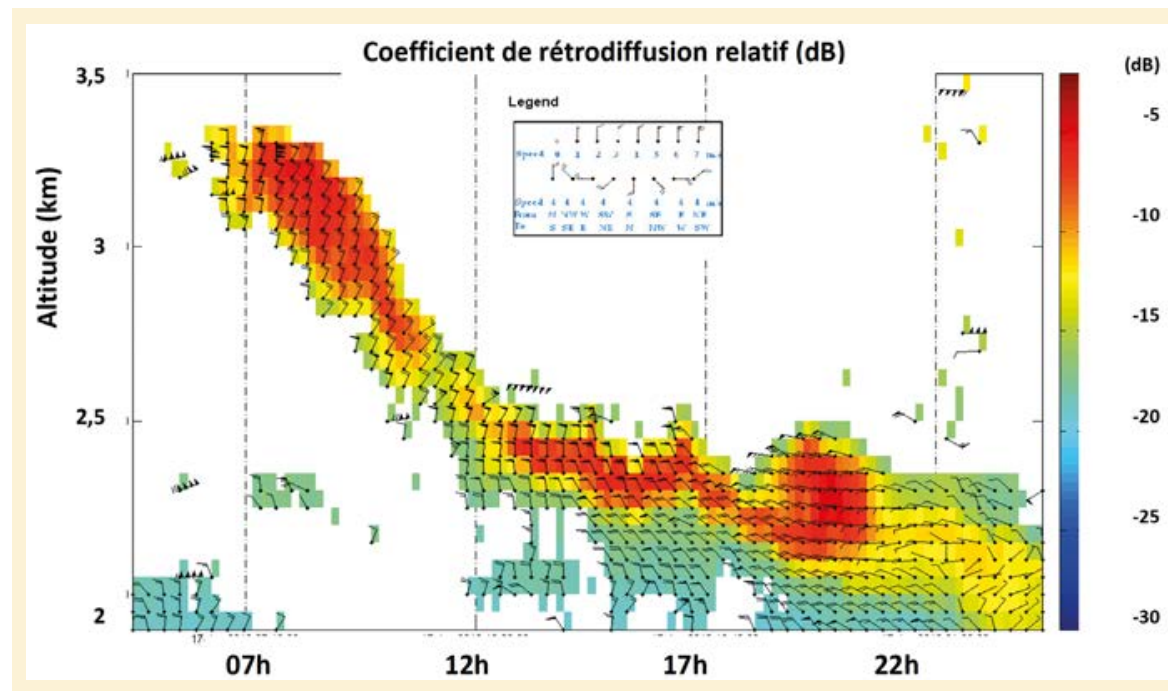

Figure 6. Nuage volcanique observé par un lidar Doppler le 17 avril 2010 (coefficient de rétrodiffusion relatif atténué superposé au champ de vent horizontal). cendres lors de son passage. La figure 6 indique la séquence de mesures du coefficient de rétrodiffusion relatif atténué, avec en superposition le champ de vent horizontal, permettant d'affiner la trajectographie du nuage. Ces instruments opérationnels participent maintenant à la surveillance des évènements volcaniques autour de Reykjavik.

\section{Une aide à la décision}

Les lidars atmosphériques contribuent à combler un manque dans la mesure et la classification des aérosols à partir du sol. Adaptés aux futurs réseaux de stations météos, ils fournissent automatiquement et en temps réel la détection et l'altitude des structures atmosphériques, les paramètres optiques de diffusion et d'extinction des aérosols, et une classification du type d'aérosols et de nuages. Leur rôle est d'améliorer la connaissance de l'impact des aérosols sur le climat et la santé humaine, et d'apporter une aide à la décision lors d'épisodes de dangers atmosphériques, comme les éruptions volcaniques.

\section{POUR EN SAVOIR PLUS}

[1] IPCC, 2014: Climate Change 2014: Synthesis Report. Contribution of Working Groups I, II and III to the Fifth Assessment Report of the Intergovernmental Panel on Climate Change [Core Writing Team, R.K. Pachauri and L.A. Meyer (eds.)]. IPCC, Geneva, Switzerland, 151 pp.

[2] J.D. Klett, LIDAR inversions with variable backscatter/extinction values, App. Opt. 24, 16381643,1985

[3] V.R. Morris, HA Winston, Laser Ceilometer CL51 Demonstration Field Campaign Report, ARM, May 2016, DOE/SC-ARM-14-042

[4] https://www.researchgate.net/publication/317256826_Assessment_of_Mixed-Layer_ HeightEstimation_from_Single-wavelength_Ceilometer_Profiles

[5] S.P. Burton, R.A. Ferrare, C.A. Hostetler, J.W. Hair, R.R. Rogers, M.D. Obland, C.F. Butler, A.L. Cook, D.B. Harper, K. D. Froyd, Aerosol classification using airborne High Spectral Resolution Lidar measurements - methodology and examples, Atmos. Meas. Tech. Discuss. 4, $5631-5688,2011$

[6] Z. Wang, K. Sassen, Cloud type and macrophysical property retrieval using multiple remote sensors, J. Appl. Meteorol. 40, 1665-1682, 2001

[7] J.C. Raut, P. Chazette, Retrieval of aerosol complex refractive index from a synergy between LIDAR, sunphotometer and in situ measurements during LISAIR experiment, Atmos. Chem. Phys. 7, 2797-2815, 2007 